\title{
Transitive inference in pigeons: Control for differential value transfer
}

\author{
JANICE E. WEAVER \\ University of Kentucky, Lexington, Kentucky \\ JANICE N. STEIRN \\ Georgia Southern University, Statesboro, Georgia \\ and \\ THOMAS R. ZENTALL \\ University of Kentucky, Lexington, Kentucky
}

\begin{abstract}
Transitive inference (TI) effects have been demonstrated in several nonhuman species using a nonverbal version of the task in which $A$ is better than $B$ is represented in a simple simultaneous discrimination, $\mathrm{A}+\mathrm{B}-$. Following five-term training (e.g., $\mathrm{A}+\mathrm{B}-, \mathrm{B}+\mathrm{C}-, \mathrm{C}+\mathrm{D}-, \mathrm{D}+\mathrm{E}-$ ), the choice of $B$ over D on test trials is taken as evidence of TI. Recently, differential value transfer from the $\mathrm{S}+$ to the $\mathrm{S}$ - in a simultaneous discrimination has been proposed as a noncognitive basis for these effects. Two experiments were conducted to control for differential value transfer. The results suggest that differential value transfer is not the only basis for nonhuman TI performance. An alternative account based on spatial mapping is discussed.
\end{abstract}

In a transitive inference (TI) task, a subject is presented pairs of stimuli that bear a particular relationship to one another and TI is said to develop when the subject can infer the relationship between novel pairings of stimuli in that set. For example, if one is told that Bill is taller than Dave and that Dave is taller than Jack, one can logically reason that Bill is taller than Jack.

In the present article, the term transitive inference is used to describe training procedures from which an ordering of stimuli might be inferred and to describe test findings that are consistent with the relational learning attributed to humans who make correct inferences on the basis of such training. The term is not intended to imply how animals perform this task.

A modified, nonverbal version of this procedure has been developed for use with nonhuman animals. In this procedure, arbitrary stimuli are presented in a series of interrelated simultaneous discriminations. In a simultaneous discrimination, two stimuli are presented together, and the choice of one stimulus $(\mathrm{S}+)$ is reinforced, whereas choice of the other stimulus $(\mathrm{S}-)$ is not reinforced. The discriminations are said to be interrelated, because the $\mathrm{S}-$ stimulus in one discrimination may serve as the $\mathrm{S}+$ stimulus in another discrimination. To adequately assess TI, a subject should be trained with a minimum of five stimuli, identified as $\mathrm{A}, \mathrm{B}, \mathrm{C}, \mathrm{D}$, and $\mathrm{E}$ for descriptive purposes, involving four discriminations: $\mathrm{A}+\mathrm{B}-, \mathrm{B}+\mathrm{C}-$,

This research was supported by National Science Foundation Grant BNS-9019080 and National Institute of Mental Health Grant MH45979, both to T.R.Z. Correspondence concerning this article should be addressed to J. E. Weaver, Department of Psychology, University of Kentucky, Lexington, KY 40506 (e-mail: jeweav1@ukcc.uky.edu).
$\mathrm{C}+\mathrm{D}-$, and $\mathrm{D}+\mathrm{E}-$. The minimum of five stimuli ensures that there is a novel test pair (BD) that does not involve a stimulus that has served only as an $\mathrm{S}+(\mathrm{A})$ or only as an $\mathrm{S}-(\mathrm{E})$. Following such training, no inherent simple reinforcement-based differential preference should have developed between $B$ and $D$, because each one was avoided in one discrimination (i.e., $\mathrm{A}+\mathrm{B}-$ and $\mathrm{C}+\mathrm{D}-$ ) and was selected in another (i.e., $\mathrm{B}+\mathrm{C}-$ and $\mathrm{D}+\mathrm{E}-$ ). Results consistent with TI have been reported in a number of nonhuman species, including squirrel monkeys (McGonigle \& Chalmers, 1977), chimpanzees (Boysen, Berntson, Shreyer, \& Quigley, 1993; Gillan, 1981), pigeons (Fersen, Wynne, Delius, \& Staddon, 1991; Steirn, Weaver, \& Zentall, 1995), and rats (Davis, 1992).

It has been proposed (Trabasso \& Riley, 1975) that findings of TI (i.e., a consistent preference for B over D in the design described) suggest relational learning or a hierarchial ordering of stimuli (i.e., $A>B>C>D>E$, where " $>$ " indicates "better than"). Trabasso and Riley suggested that subjects form a mental representation which is a linear mapping of the stimuli that preserves and organizes all the essential information required for subjects to correctly choose B over $\mathrm{D}$, on a BD test.

On the other hand, two noncognitive accounts of findings of TI with such a design have been offered to explain TI performance in animals. According to one of these, differences in reinforced and nonreinforced experiences with the test stimuli themselves are responsible for TI performance (Couvillon \& Bitterman, 1992). Although nominally the reinforcement histories associated with the test stimuli are equated, it has been suggested that there are likely to be fewer nonreinforced responses to $\mathrm{B}$ in the presence of $\mathrm{A}$ (to which responses are always 
reinforced) than nonreinforced responses to $D$ in the presence of $\mathrm{C}$ (to which responses are not always reinforcedi.e., in the presence of B; see Wynne, Fersen, \& Staddon, 1992). Thus, if $D$ has more of a history of nonreinforcement than B has, then B should be preferred over D. It should be noted that Couvillon and Bitterman's (1992) hypothesis focused on nonreinforcement histories of $B$ and $D$ because they were attempting to account for findings by Fersen et al. (1991), who used a correction procedure that ensured an equal number of reinforcements to all the $\mathrm{S}+\mathrm{s}$. If a correction procedure were not employed, any differences in reinforced responding between $\mathrm{B}$ and $D$ would also be relevant.

To test the reinforcement history hypothesis, Steirn et al. (1995) examined the relative reinforcement and nonreinforcement experiences that pigeons had with the test stimuli in their TI experiment. To assess the contributions that reinforcement history might have on preference for B over D in test, they calculated ratios for the relative reinforcement history associated with $\mathrm{B}$ [reinforced choices of $B /(B+D)]$ in training and the relative nonreinforcement history associated with $\mathrm{D}$ [nonreinforced choices of $D /(D+B)$ ] in training. If reinforcement history makes a difference, either or both of these ratios should have been significantly greater than .50 . Neither was. Furthermore, neither ratio was positively correlated with preference for B over D (see also Zentall \& Sherburne, 1994).

Fersen et al. (1991) suggested an alternative noncognitive mechanism to account for the TI results of nonhumans. According to this view, positive value is transferred (indirectly) from the reinforced member of each stimulus pair to the nonreinforced member of the pair, in proportion to the direct value of the $S+$. Thus in the pair $\mathrm{A}+\mathrm{B}-, \mathrm{B}$ acquires some positive value because it is presented with $A$. This transferred value from $A$ is added to any value that $\mathrm{B}$ acquires directly from reinforced responding in the presence of another stimulus (i.e., $\mathrm{C}$ in the TI procedure). Thus, although the direct values of $B$ and $\mathrm{D}$ are comparable, value transfer theory (VTT) predicts a preference for $\mathrm{B}$ over $\mathrm{D}$ following training on the five-element series, because $B$ receives additional value from a highly valued stimulus (A) to which responding has been always reinforced, whereas $D$ receives additional value from a less valued stimulus $(\mathrm{C})$ to which responding has been both nonreinforced $(\mathrm{B}+\mathrm{C}-)$ and reinforced $(\mathrm{C}+\mathrm{D}-)$.

Zentall and Sherburne (1994) tested for evidence of value transfer, under conditions that precluded TI. They presented pigeons with two pairs of stimuli $\mathrm{A}+\mathrm{B}-$ and $C \pm D-$, in which responses to $A$ were always reinforced, responses to $C$ were reinforced $50 \%$ of the time, and responses to B and D were never reinforced. Zentall and Sherburne found, consistent with VTT, that pigeons preferred B over D on BD test trials. In a similar vein, Steirn et al. (1995, Experiment 3) tested VTT by training pigeons on a series of simultaneous discrimination pairs even more like those used in previous TI research with animals, in which it was not possible to arrange the train- ing pairs in a logical linear order (the pairs were $\mathrm{A}+\mathrm{B}-$, $\mathrm{C}-\mathrm{E}+, \mathrm{C}+\mathrm{D}-$, and $\mathrm{A}+\mathrm{E}-$ ). According to VTT, although there was no linear ordering to the stimuli, the training should have resulted in differential value transfer similar to that proposed to occur in a TI task, because B was presented with a stimulus (A) to which responding had been always reinforced, whereas $D$ was presented with a stimulus (C) to which responding had not always been reinforced. As predicted from VTT, when pigeons were given a $\mathrm{BD}$ test, they consistently preferred B over D.

Although VTT has received empirical support independent of the TI task, the presence of differentially transferred value does not rule out the possibility of alternative accounts of the TI effect. In the VT studies discussed above, a transitive relation among the elements was not possible. Although it appears that transitive relations are not needed to account for these results, the results of these studies do not eliminate the possibility that transitive relations could contribute to the basis of choice on BD test trials in the typical TI task with animals.

Weaver (1994) examined TI while attempting to equate for presumed differential positive value transfer by making the value of $\mathrm{A}$ in training more similar to the value of $C$. This manipulation involved reinforcing a random $50 \%$ of the choices of A while responses to B continued to be nonreinforced. According to VTT, a reduction in the direct value of A should make it more similar in value to $\mathrm{C}$ and should thus reduce or eliminate the preference for $B$ over D. Contrary to that expectation, reducing the value of A produced little decrement in choice of B over D. Pigeons continued to choose B over D on $76.0 \%$ of their test trials, a level comparable to that of a standard group in which responses to $\mathrm{A}$ were always reinforced $(77.2 \%$ choice of B over D). This finding was replicated by Steirn and Weaver (1995), who found that pigeons for which $50 \%$ of their choices of $\mathrm{A}$ in training were reinforced chose B $(65.8 \%)$ on BD test trials at a level comparable to that of pigeons reinforced for all choices of $A$ in training $(69.3 \%)$.

The results of these experiments suggest that attempts to equate for value transfer have had little effect on the magnitude of the TI effect found. Thus, it appears that positive value transfer may not be the only basis of test preference in the TI task. One should note, however, that in TI training not only is B typically paired with the always positive $A$, but also $D$ is paired with the always negative E. Therefore, it is possible that negative value transfer could contribute to the TI effect found by Weaver (1994). If $D$ has more negative value transferred to it from $E$ than $B$ has transferred to it from $C$, the relatively greater avoidance of D could account for the TI effects typically reported.

Steirn and Weaver (1995) attempted to manipulate the value of $\mathrm{E}$ after original TI training and found that it had no effect on preference for B over D in test, but the manipulation involved presenting the $\mathrm{E}$ stimulus alone rather than in the context of a simultaneous discrimination with D. Thus, negative value transfer may still play a role in 
TI effects that have been found with the five-term series procedure. The purpose of Experiment 1 was to test for the effects of transitive inference under conditions in which we attempted to equate for the effects of both positive and negative value transfer on the test stimuli.

\section{EXPERIMENT 1}

In an attempt to equate for both positive and negative value transfer, pigeons in Experiment 1 were trained with responses to both $\mathrm{A}$ and $\mathrm{E}$ reinforced on a random $50 \%$ of the trials in which they were chosen (Group E50). Choices of $B$ versus $D$ were then compared with those of a control group for which choices of A were reinforced a random $50 \%$ of the time in training and choices of $E$ were never reinforced (Group E0).

\section{Method}

Subjects. The subjects were 8 experimentally naive, mixed-sex White Carneaux pigeons (Columba livia) acquired as retired breeders (7-9 years old) from the Palmetto Pigeon Plant (Sumter, SC). The pigeons were housed individually with free access to grit and water in a colony room on a 12:12-h light:dark cycle and were maintained at $80 \%$ of their free feeding body weights.

Apparatus. The apparatus was a pigeon operant chamber manufactured by BRS/LVE (Laurel, MD). The inside of the chamber measured $35 \mathrm{~cm}$ high, $30 \mathrm{~cm}$ from the front panel to the back wall, and $36 \mathrm{~cm}$ across the front panel. Mounted on the front panel were three square response keys, each $3 \mathrm{~cm}$ high $\times 3 \mathrm{~cm}$ wide, spaced $1 \mathrm{~cm}$ apart. The tops of the keys were located $8 \mathrm{~cm}$ from the top of the chamber. In this experiment, only the center and right keys were used. Mounted behind each response key was a 12-stimulus inline projector that projected onto the keys the stimuli (red, R; yellow, Y; white, W; blue, B; and green, G) produced by Kodak Wratten Filters (Nos. 26 and 9, no filter and Nos. 38a and 60, respectively). The pigeons' received access to mixed grain reinforcement (Purina Pro Pigeon Grains) through an aperture $(5.5 \mathrm{~cm}$ wide, $5 \mathrm{~cm}$ high), the bottom of which was centered $9 \mathrm{~cm}$ from the grid floor. The aperture was illuminated during reinforcement. White noise and an exhaust fan masked extraneous noise. General illumination was provided by a houselight, the bottom of which was centered $5.5 \mathrm{~cm}$ above the top of the center key. A microcomputer located in an adjacent cubicle controlled the sessions and recorded data.

Procedure. Following magazine training, all pigeons were trained by successive approximations to peck the center and right keys. Each of the five stimuli appeared at random twice on each key during the 20 pretraining trials. The trials were separated by $10-\mathrm{sec}$ intertrial intervals (ITIs). Reinforcement occurred during the first $1.5 \mathrm{sec}$ of the ITI on each of the three shaping sessions. In the first shaping session, the houselight was on and one response to the stimulus was required for reinforcement (fixed ratio, FRI). During the second session, five responses were required for reinforcement (FR5). The third session was the same as the second, except that the houselight was lit only during the ITI.

The pigeons were randomly assigned to two groups. The stimuli $A$, $B, C, D$, and $E$ were represented by $R, Y, W, B$, and G, respectively, for half the pigeons in each group, and by $\mathrm{G}, \mathrm{B}, \mathrm{W}, \mathrm{Y}$, and $\mathrm{R}$, respectively, for the remaining pigeons. Training involved four phases, one for each stimulus pair. In Phase 1, the first premise pair (AB) was presented as a simultaneous discrimination (on the center and right keys). Five consecutive pecks to either stimulus constituted a choice. A choice resulted in the termination of both stimuli and the onset of the $10-\mathrm{sec}$ ITI, with the chamber illuminated by the houselight. For all pigeons, the choice of stimulus $A$ resulted in access to reinforcement on a random half of the trials. For the remaining half of the trials on which $A$ was chosen and for all trials on which B was chosen, a choice was immediately followed by the ITI and no food was available. The position of the correct stimulus in each pair was balanced over the 96 trials of
Table 1

Designs of Experiments 1 and 2

\begin{tabular}{cccccc}
\hline & & \multicolumn{4}{c}{ Phase } \\
\cline { 3 - 6 } Experiment & Group & 1 & 2 & 3 & 4 \\
\hline 1 & E50 & $\mathrm{A} \pm \mathrm{B}-$ & $\mathrm{B}+\mathrm{C}-$ & $\mathrm{C}+\mathrm{D}-$ & $\mathrm{D}+\mathrm{E} \pm$ \\
& $\mathrm{E} 0$ & $\mathrm{~A} \pm \mathrm{B}-$ & $\mathrm{B}+\mathrm{C}-$ & $\mathrm{C}+\mathrm{D}-$ & $\mathrm{D}+\mathrm{E}-$ \\
2 & $\mathrm{ACE} 50$ & $\mathrm{~A} \pm \mathrm{B}-$ & $\mathrm{B}+\mathrm{C} \pm$ & $\mathrm{C} \pm \mathrm{D}-$ & $\mathrm{D}+\mathrm{E} \pm$ \\
\hline
\end{tabular}

Note-“+" denotes $100 \%$ reinforcement for choice, " \pm " denotes $50 \%$ reinforcement for choice, and "-" denotes nonreinforcement for choice.

each session, with the restriction that on no more than 3 consecutive trials could the stimuli occur in the same position. Phase 1 continued for a minimum of three sessions and until $A$ was selected on at least $90 \%$ of the trials for two consecutive sessions. Meeting these criteria advanced the pigeon to the next phase with the next premise pair (i.e., $\mathrm{BC}$ with $\mathrm{B}$ defined as correct) on the following session. Phases 2,3 , and 4 proceeded in the same manner, with the exceptions that for both groups the correct choice in the pair always resulted in reinforcement and in Phase 4 incorrect choices of $E$ were reinforced on a random $50 \%$ of trials for Group E50. The stimulus pairs presented and reinforcement conditions available in the four phases are presented in Table 1. Sessions occurred once a day, 6 days per week. After completion of Phase 4 training, each subject received a single session of 24 trials with the novel test pair BD. Choice of either stimulus was reinforced on a random half of the test trials.

\section{Results and Discussion}

Acquisition. For Group E0, the mean sessions to acquire the discriminations in Phases 1-4 were 2.8, 2.5, 3.0, and 2.2, respectively. For Group E50, the mean sessions required to acquire those discriminations were $2.8,2.8$, 2.5 , and 3.2 , respectively. Acquisition data were analyzed with a two-way mixed factors analysis of variance (ANOVA) with group and phase as factors. The .05 level of significance was employed in these and all subsequent analyses. The analysis indicated that there was no significant effect of group $(F<1)$ or phase $(F<1)$, nor was there a significant group $\times$ phase interaction $[F(3,18)=$ 1.8]. The mean number of correct and incorrect choices of each stimulus is reported in Table 2 .

According to reinforcement history models of TI, test performance can be predicted from the reinforcements and nonreinforcements of the test stimuli in training. Precise predictions of preference based on reinforcement history models require the estimation of parameter values and depend on the exact sequence of reinforced and nonreinforced responses to the stimuli (see Couvillon \& Bitterman, 1992; Steirn et al., 1995; Wynne et al., 1992). According to a general reinforcement history account, however, it should be the case that choice of B over D results from more reinforced responses to $B$ than to $D$, or conversely from more nonreinforced responses to $\mathrm{D}$ than to $B$. To evaluate this hypothesis, two ratios were computed for each bird. The reinforcement history of B was calculated as the proportion of reinforced choices of $B$ (in the $\mathrm{B}+\mathrm{C}-$ discrimination) to the total reinforced choices of $\mathrm{B}$ and $\mathrm{D}$ (in the $\mathrm{D}+\mathrm{E} \pm$ or $\mathrm{D}+\mathrm{E}-$ discrimination for groups E50 and E0, respectively) in training. Similarly, the nonreinforcement history of $\mathrm{D}$ was calculated as the proportion of nonreinforced choices of D (in the $\mathrm{C}+\mathrm{D}-$ discrimination) to the total nonreinforced 
Table 2

Mean Number of Correct and Incorrect Choices of Each Stimulus in Training for Groups E0, E50, and ACE50

\begin{tabular}{lccr}
\hline \multirow{2}{*}{ Group } & & \multicolumn{2}{c}{ No. Choices } \\
\hline E0 & Stimulus & Correct & Incorrect \\
& A & 267.2 & \\
& B & 272.8 & 20.8 \\
& C & 268.5 & 15.2 \\
E50 & D & 278.2 & 43.5 \\
& E & & 9.8 \\
& A & 289.0 & \\
& B & 253.2 & 23.0 \\
ACE50 & C & 268.5 & 34.8 \\
& D & 268.2 & 19.5 \\
& E & & 43.8 \\
& A & 303.4 & 80.6 \\
& B & 289.4 & 70.6 \\
& C & 280.4 & 31.6 \\
& D & 346.9 & 121.1 \\
\hline
\end{tabular}

choices of $B$ (in the $A \pm B-$ discrimination) and $D$ in training. According to a reinforcement history account, to explain a significant preference for $B$ over $D$, either or both of these ratios should be significantly greater than .50. The ratios for reinforcement (.49) and nonreinforcement (.56) were not significantly different from .50, however $[F(1,7)=1.47$ and $F<1$, respectively]. Thus, according to a reinforcement history account there should be no basis for a TI effect in the present experiment.

Test. Mean percentage choice of $B$ over $D$ for Group E0 was 73.0, and for Group E50, 78.0. A two-way mixed ANOVA confirmed that choice of B was significantly greater than chance overall $[F(1,6)=10.15]$, and neither the difference between groups, nor the group $\times$ choice of $\mathrm{B}$ interaction was significant (both $F_{\mathrm{S}}<1$ ). In fact, the pigeons in Group E50 chose B over D slightly more than did pigeons in Group E0. Thus, choice of B over D appeared not to be affected by the probability of reinforcement associated with $E$ in training.

\section{EXPERIMENT 2}

In Experiment 1 (as well as in Steirn \& Weaver, 1995, and Weaver, 1994), the reinforcement value of $A$ was reduced to $50 \%$ in an attempt to equate the (direct) values of $A$ and $C$ (the stimuli presumed to transfer value to $B$ and $D$, respectively). In that experiment, it was assumed that the value of a stimulus associated with random $50 \%$ reinforcement (A) was comparable to that of a stimulus to which responses were not reinforced in one discrimination $(\mathrm{B}+\mathrm{C}-)$ but were reinforced in the following discrimination $(\mathrm{C}+\mathrm{D}-)$. A similar assumption was made for the relation between stimuli $\mathrm{C}$ and $\mathrm{E}$ (to which responses were reinforced $50 \%$ of the time in Group E50). The purpose of Experiment 2 was to determine whether TI performance would be found when there was random $(50 \%)$ reinforcement for choices of $A, C$, and $E$ in all phases of training. That is, would TI be found when sim- ilar patterns of reinforcement were associated with $\mathrm{A}, \mathrm{C}$, and $\mathrm{E}$ (the stimuli that according to VTT are presumed to transfer differential positive or negative values to $B$ and $\mathrm{D}$ in training).

\section{Method \\ Subjects. The subjects were 8 experimentally naive pigeons. They were similar in all respects to those used in Experiment 1. \\ Apparatus. The apparatus was the same as that used in Experi- ment 1 . \\ Procedure. The procedure for all pigeons was the same as that used for Group E50 in Experiment 1, except that choices of $\mathrm{C}$ were rein- forced on a random $50 \%$ of the trials in both Phases 2 and 3 . Thus, all 8 pigeons acquired the discriminations $\mathrm{A} \pm \mathrm{B}-, \mathrm{B}+\mathrm{C} \pm, \mathrm{C} \pm \mathrm{D}-$, and $\mathrm{D}+\mathrm{E} \pm$ in Phases 1-4, respectively (see Table 1 for comparison of this design with that used in Experiment 1).}

\section{Results and Discussion}

Acquisition. The mean number of sessions required to reach criterion was 3.81 . The mean sessions to acquire Phases 1-4 were $4.0,3.5,2.8$, and 4.9 , respectively. In Experiment 2, sessions to criterion did differ significantly as a function of phase $[F(3,21)=4.36]$. The critical comparisons were those between phases in which choices of $B$ and $D$ were reinforced (Phase 1 vs. Phase 3 ) and between phases in which choices of $B$ and $D$ were nonreinforced (Phase 2 vs. Phase 4). Planned comparisons revealed that sessions to acquire the discrimination in Phase 2 did not differ significantly from those of Phase $4[F(1,7)=3.32]$; however, the pigeons required significantly fewer sessions to reach criterion in Phase 3 than they did in Phase $1[F(1,7)=5.64]$. The difference in nonreinforcement history associated with Phases 1 and 3 indicates that there were more incorrect choices on the $\mathrm{A} \pm \mathrm{B}$ - discrimination than on the $\mathrm{C} \pm \mathrm{D}$ - discrimination. Thus, any effect of this difference in nonreinforcement history should be in the opposite direction of that predicted by the TI effect (choice of B over D).

As in Experiment 1, the reinforcement and nonreinforcement ratios were examined (see Table 2 for the mean numbers of correct and incorrect choices of each stimulus). The reinforcement ratio [reinforced responses to $\mathrm{B} /(\mathrm{B}+\mathrm{D})]$ was .46 , which is not significantly different from . $50[F(1,7)=1.43]$. The nonreinforcement ratio [nonreinforced responses to $\mathrm{D} /(\mathrm{B}+\mathrm{D})$ ] was .31 , which was significantly below chance $[F(1,7)=10.12]$. The nonreinforcement ratio indicates that there were actually relatively more nonreinforced choices of $B$ than of $D$. Thus, the differential nonreinforcement history associated with the test stimuli would predict a preference for D over B in test. This prediction is in a direction opposite that of a TI effect.

Test. Once again, mean percent choice of $B$ in test (68.9) was significantly greater than chance $[50 \% ; F(1,7)=$ 6.94] and was comparable to the respective values obtained in Experiment 1. Since responses to A, C, and E were all reinforced on a random $50 \%$ of the trials in which they were chosen, according to VTT differential value (either positive or negative) should not have transferred to the two test stimuli. 


\section{GENERAL DISCUSSION}

In two experiments, manipulations that, according to VTT, should have reduced or eliminated differential value transfer to the test stimuli failed to affect the magnitude of TI effects. These results are consistent with the earlier finding that test preference for B over D did not differ as a function of either the value ( $100 \%$ vs. $50 \%$ reinforcement) of A (the stimulus paired with B in training; Steirn \& Weaver, 1995; Weaver, 1994) or that of E after training (but before the BD test; Steirn \& Weaver, 1995). Furthermore, in Experiment 2 of the present study, even when all the stimuli that had been paired with the test stimuli in training should have had comparable value, strong evidence for TI was found. In addition, although not directly manipulated, in both experiments the reinforcement and nonreinforcement experiences with the $B$ and D stimuli did not appear to predict the preference for B over D found in test. Thus, it seems unlikely that either differential direct reinforcement value associated with the test stimuli in training or the differential value transferred to the test stimuli in training serves as the basis of test stimulus preference.

Another possible interpretation arises from the nature of the procedure of the present study. In the present procedure (as well as that used by Davis, 1992; and Steirn et al., 1995), a sequential design was used during training. In a sequential design, all training involving a single stimulus pair occurs within a block of trials. Steirn et al, and others have suggested that with sequential training, because the test stimuli (B and D) are not trained at the same time, it is possible that the test stimulus that is avoided last in training (i.e., D) is most likely to be avoided on test trials. To determine whether a recency bias could explain the animals' TI preference in test, when training was sequential, Davis (1992) and Steirn et al. reversed the order in which pairs were trained prior to test (i.e., $\mathrm{E}-\mathrm{D}+$, followed by $\mathrm{D}+\mathrm{C}-$, followed by $\mathrm{C}-\mathrm{B}+$, followed by $\mathrm{B}-\mathrm{A}+$ ) and found a level of TI performance comparable to that obtained with the other training order. Thus, TI test results using a sequential training procedure cannot be attributed to a recency bias.

Although the present research casts doubt on both value transfer and reinforcement history as the basis of $\mathrm{Tl}$ effects in animals, the issue of what mechanism underlies nonhuman TI performance remains unclear. The possibility that rats and pigeons are demonstrating propositional logic seems most unlikely. Indeed, Piaget (1970) suggests that children younger than 7 years old lack the cognitive abilities necessary to correctly perform TI tasks. Although Bryant and Trabasso (1971) have demonstrated TI effects in children as young as 4 years old, it remains likely that young children and animals utilize a simpler mechanism that leads to a similar result.

One mechanism, first proposed for young children by Trabasso and Riley (1975), may be the formation of spatial representations, or a mental mapping, of the stimuli according to their relative position in a series. That such a process underlies animals' TI performance is supported by research by Roberts and Phelps (1994), who trained rats on a spatial TI task in which the premise pairs were presented in either a linear or a nonlinear configuration (i.e., the correct response in training was always confounded with a relative spatial position). In the linear configuration condition, the stimulus pairs were presented at a location consistent with their positions in a hypothetical linear display. It was suggested that training with the linear configuration procedure might facilitate the formation of a linear spatial representation, which in turn would facilitate TI performance, whereas training with the nonlinear configuration procedure would not.

Rats trained with the linear configuration condition demonstrated a significant $\mathrm{Tl}$ effect $(73.4 \%$ choice of $\mathrm{B}$ over $\mathrm{D})$ when given a $\mathrm{BD}$ test in a different context (Y-maze) with spatial location of the two test stimuli varied, whereas those trained with a nonlinear presentation did not ( $47.9 \%$ choice of B). Roberts and Phelps's (1994) results suggest that training with a redundant spatial cue can facilitate the spatial representation of the stimulus pairs by rats, in spite of the fact that those spatial cues were irrelevant on test trials. The establishment of linear representations may also be the basis for TI effects found in young children and animals. Such a solution would suggest that organisms "translate" the trained stimulus relations into a single (spatial) "list," with each item's position in the list determined by stimuli, common to two pairs, that can serve as mediators. Research of the type reported by Roberts and Phelps may help to clarify the nature of the representation of the training stimuli experienced by animals in a TI task.

\section{REFERENCES}

Boysen, S. T., Berntson, G. G., Shreyer, T. A., \& Quigley, K. S. (1993). Processing of ordinality and transitivity in chimpanzees (Pan troglodytes). Journal of Comparative Psychology, 107, 208-215.

Bryant, P. E., \& Trabasso, T. (1971). Transitive inferences and memory in young children. Nature, 232, 456-458.

Couvillon, P. A., \& Bitterman, M. E. (1992). A conventional conditioning analysis of "transitive inference" in pigeons. Journal of Experimental Psychology: Animal Behavior Processes, 18, 308-310.

Davis, H. (1992). Transitive inference in rats (Rattus norvegicus). Journal of Comparative Psychology, 106, 342-349.

Fersen, L. von, Wynne, C. D. L., Delius, J. D., \& Staddon, J. E. R. (1991). Transitive inference formation in pigeons. Journal of Experimental Psychology: Animal Behavior Processes, 17, 334-341.

GILLAN, D. J. (1981). Reasoning in the chimpanzee: II. Transitive inference. Journal of Experimental Psychology: Animal Behavior Processes, 7, 150-164.

MCGonigle, B. O., \& Chalmers, M. (1977). Are monkeys logical? Nature, 267, 694-696.

Piaget, J. (1970). Genetic epistemology (E. Duckworth, Trans.). New York: Columbia University Press.

Roberts, W. A., \& Phelps, M. T. (1994). Transitive inference in rats: A test of the spatial coding hypothesis. Psychological Science, $\mathbf{5}$, 368-374

Steirn, J. N., \& Weaver, J. E. (1995, May). Positive and negative value transfers in a transitive inference task with pigeons. Paper presented at the Midwestern Psychological Association Conference, Chicago.

Steirn, J. N., Weaver, J. E., \& Zentall, T. R. (1995). Transitive inference in pigeons: Simplified procedures and a test of value transfer theory. Animal Learning \& Behavior, 23, 76-82.

Trabasso, T., \& Riley, C. A. (1975). The construction and use of representations involving linear order. In R. L. Solso (Ed.), Information processing and cognition: The Loyola Symposium (pp. 381-410). Hillsdale, NJ: Erlbaum.

WEAVER, J. E. (1994). An examination of mechanisms underlying transitive inference performance by pigeons: Linear order theory vs. value transfer theory. Unpublished master's thesis, Georgia Southern University.

Wynne, C. D. L., Fersen, L. von, \& Staddon, J. E. R. (1992). Pigeons' inferences are transitive and the outcome of elementary conditioning principles: A response. Journal of Experimental Psychologv: Animal Behavior Processes, 18, 313-315.

Zentall, T. R., \& Sherburne, L. M. (1994). Transfer of value from $\mathrm{S}+$ to $\mathrm{S}-$ in a simultaneous discrimination. Journal of Experimental Psychology: Animal Behavior Processes, 20, 176-183.

(Manuscript received March 10, 1996; revision accepted for publication October $7,1996$. 